\title{
APORTES DE LAS UNIVERSIDADES PÚBLICAS EN TORNO AL HOSTIGAMIENTO SEXUAL EN COSTA RICA
}

\section{THE CONTRIBUTIONS OF PUBLIC UNIVERSITIES AGAINST SEXUAL HARASSMENT IN COSTA RICA}

\author{
Doris Fernández Carvajal*
}

RESUMEN

Desde el Instituto de Estudios de la Mujer de la Universidad Nacional de Costa Rica, se está realizando una investigación denominada "Debates sobre salud sexual y salud reproductiva en Costa Rica" (código 0056-18). Uno de los temas de debate en los últimos cincuenta años es el hostigamiento sexual, el cual ha generado un interés investigativo en las universidades públicas del país a través de diversos trabajos. Para la elaboración de este artículo se revisó un total de 23 trabajos, la mayor parte eran tesis de licenciatura y de maestría. El artículo presenta una descripción de los resultados de esas investigaciones. Estos trabajos han aportado al conocimiento de una problemática social muy arraigada en nuestra sociedad. De igual manera, han generado una conciencia crítica sobre este tipo de violencia y, a la vez, han contribuido a impulsar acciones para desestimular y sancionar estas prácticas indeseadas en los ámbitos de estudio y trabajo.

PALABRAS CLAVE: COSTA RICA * DISCRIMINACIÓN * ACOSO SEXUAL * VIOLENCIA * UNIVERSIDAD

\section{ABSTRACT}

The Women Studies Institute of the National University of Costa Rica is carrying out a study called "Debates on Sexual and Reproductive Health in Costa Rica" (code 005618). Sexual harassment has been one of the topics that has stir a lot of debate in the last fifty years and it has generated research interest within the country's public universities through various theses and dissertations. For the preparation of this article, a total of 23 works were reviewed, most of them were undergraduate and master's thesis. The article presents a description of the results of these investigations. These works have contributed to the knowledge of a deeply rooted social problem in our society. In the same way, they have generated a critical awareness about this type of violence, while they have contributed to promoting actions to discourage and punish these unwanted practices in workplaces and in learning environments.

KEYWORDS: COSTA RICA * DISCRIMINATION * SEXUAL ABUSE * VIOLENCE * UNIVERSITY

* Instituto de Estudios de la Mujer, Universidad Nacional, Costa Rica. doris.fernandez.carvajal@una.cr 


\section{INTRODUCCIÓN}

Desde el Instituto de Estudios de la Mujer de la Universidad Nacional de Costa Rica, se está realizando una investigación denominada Debates sobre salud sexual y salud reproductiva en Costa Rica. Este trabajo pretende analizar los ejes sobre los cuales ha girado la discusión en el país, en relación con la sexualidad en los últimos 50 años, es decir, a partir de la década de los años 70 hasta el presente. Algunas de estas discusiones han girado en torno a la educación en sexualidad, las enfermedades de transmisión sexual y la planificación familiar. En esta misma línea, uno de los temas que aparece en la escena nacional en la década de los 90 es el hostigamiento sexual, por ese motivo, el presente artículo recoge los resultados de investigaciones realizadas desde tres universidades públicas del país. Estos trabajos han sido de gran utilidad para visibilizar una problemática social muy arraigada en nuestra sociedad.

La Universidad de Costa Rica (UCR) es la que registra el mayor número de trabajos, seguida por la Universidad Nacional (UNA) y la Universidad Estatal a Distancia (UNED). Cabe señalar que el Instituto Tecnológico de Costa Rica (TEC) no presenta investigaciones de este tipo, ya que su oferta académica, centrada en la tecnología, no ha despertado el interés en este objeto de estudio, aunque es importante mencionar que la Oficina de Equidad de Género ha llevado a cabo algunos sondeos para conocer la incidencia del fenómeno en ese espacio de estudio.

Los resultados de estas investigaciones también han servido de base para la realización de acciones dirigidas a evidenciar, informar, sensibilizar y a crear conciencia en las personas trabajadoras $y$ en el estudiantado sobre la ocurrencia de dicho fenómeno en diversas instituciones públicas y privadas. También han sido de utilidad para mostrar quiénes son las personas que cometen el hostigamiento sexual $y$ hacia quiénes lo dirigen; de la misma forma, han expuesto los efectos negativos, tanto físicos como emocionales, $y$ otros no menos importantes, tales como, el perjuicio en los ámbitos laborales y de estudio de las víctimas. Aunado a lo anterior, han permitido identificar vacíos en la normativa y evidenciar limitaciones en la aplicación de la ley en las instituciones.

Para la elaboración de este artículo, se revisaron 23 trabajos realizados en las universidades antes mencionadas. La mayor parte de estos fueron revisados en "físico" en las bibliotecas de las respectivas universidades, mientras que un número menor se revisó en "línea". El análisis de cada trabajo se centró en el planteamiento del objeto de estudio, la población estudiada, el abordaje teórico y sus resultados. De la revisión de los trabajos realizados, se puede constatar que los proyectos de investigación tuvieron como objetivo conocer el "estado de la cuestión". Posteriormente, se revisaron las tesis de grado de diversas disciplinas: Derecho, Psicología, Trabajo Social, Enfermería y Educación, todas estas se realizaron para la obtención del título de licenciatura. Estos trabajos son importantes ya que contribuyeron a mostrar o evidenciar la ocurrencia del hostigamiento en las instituciones donde se realizó dicho estudio (escuelas, colegios, hospital, hoteles, universidades). Por su parte, en las tesis de Derecho se interesa por determinar la aplicación de los reglamentos en las instituciones, la duración de los procesos y la aplicación de las sanciones.

Finalmente, se encuentran las tesis a nivel de maestría, que de igual manera evidencian dicha problemática en espacios laborales y educativos con un abordaje teórico feminista. Estas últimas tesis se realizaron en el marco de una maestría conjunta entre la Universidad de Costa Rica y la Universidad Nacional a partir del año 1993.

En 1995, se aprueba en el país la Ley contra el hostigamiento sexual en el empleo y la docencia (nro. 7476). En este artículo, se presentan los resultados de los trabajos realizados anteriores a la aprobación de la citada Ley y los realizados posteriores a la misma.

Recientemente, se dispone de mayor conocimiento e información sobre esta problemática social, que lleva a reconocer el hostigamiento sexual como una manifestación de violencia que sufren las mujeres por su condición de género, así como una práctica discriminatoria que violenta los derechos humanos de las personas que lo sufren. Pero llegar a 
este nivel de conocimiento $y$ de conciencia ha tomado su tiempo, el hostigamiento sexual visto como un problema social, al igual que otros fenómenos sociales, ha tenido un "recorrido histórico".

\section{ALGUNOS ANTECEDENTES HISTÓRICOS SOBRE EL HOSTIGAMIENTO SEXUAL ${ }^{1}$}

En las décadas anteriores a los años 70, este fenómeno se caracterizó por estar rodeado de una especie de "ambigüedad", una de estas era ¿qué podía o no considerarse hostigamiento sexual?, ¿existían dudas sobre si estas prácticas ocurrían solamente en espacios laborales?, ¿quiénes podían ser víctimas de acoso, solo las mujeres o también los hombres?, ¿estos actos tendrían características diferentes si provenían de uno u otro sexo? Todas estas inquietudes subsistían en un momento en que se consideraba que las identidades se constituían sobre la base de la biología.

En esta misma línea, el libro de Wise y Stanley (1992) denominado El acoso sexual en la vida cotidiana, es considerado un trabajo pionero, ya que fue una de las primeras publicaciones sobre esta problemática $y$, además, es citado en la mayor parte de los trabajos que se revisaron. Este aporta algunos antecedentes históricos sobre el hostigamiento sexual. Las autoras señalan que, los primeros en sacarlo a la luz pública, fueron los medios de prensa escrita ingleses, al apelar a la preocupación de algunos sindicatos por esta situación. El interés sindical por este fenómeno era comprensible, ya que este tipo de prácticas impactaban de manera negativa el ámbito laboral. Uno de los medios llegó a definir el hostigamiento sexual como "una conducta sexual natural de ciertos hombres un tanto fuera de lo común" (Wise y Stanley, 1992, p. 40). Las ideas subyacentes sobre el hostigamiento sexual de esa época,

1 La mayor parte de los trabajos revisados utilizan la categoría hostigamiento sexual, al igual que la Ley nro. 7476, son pocos los que se refieren a acoso sexual. Sin embargo, para este artículo se utilizará la tendencia de considerarlos como sinónimos, aunque se le dará prioridad al término de hostigamiento sexual. presentaban a un hombre acosador, con un desbordado apetito sexual con incapacidad para controlarlo. Esta percepción sobre la sexualidad masculina contribuyó a trivializar el hostigamiento sexual, al considerarlo como "prácticas excepcionales" cometidas por pocos hombres.

Paralelamente a lo anterior, también se llegó a considerar el acoso como parte de situaciones de "galanteos y amoríos" en los que se veían involucrados hombres y mujeres en los espacios de trabajo. Estos fueron vistos como un "ingrediente" que ayudaba a darle un ambiente agradable al trabajo. Más adelante, las primeras encuestas dieron cuenta que mujeres inglesas manifestaron haber sido objeto de hostigamiento sexual en su lugar de trabajo.

Según Wise y Stanley (1992), en la década de los 80, es cuando comenzó a mencionarse el hostigamiento sexual en el ámbito universitario de la sociedad inglesa. Para ese entonces, aparecieron "los Romeos universitarios que acosaban sexualmente a las estudiantes, también los catedráticos lascivos, decanos lujuriosos y poco tiempo después aparecieron notas sobre los Romeos políticos, considerados como "manos largas". (p. 47).

En Estados Unidos, se encuentran dos antecedentes sobre este fenómeno, por un lado, se ha afirmado que la categoría "acoso sexual", fue utilizada por primera vez por un grupo de feministas norteamericanas que laboraban en la Universidad de Cornell, cuando se interesaron por compartir con otras mujeres, sus experiencias de trabajo con hombres. De igual manera, la publicación del libro, Sexual Harassment of working woman, en el año 1979, por Catharine MacKinnon, es considerado un referente en la materia. Sus planteamientos contribuyeron a perfilarlo como un problema social desde una perspectiva feminista, al sostener que el hostigamiento sexual es una expresión del control masculino sobre la sexualidad femenina. Al mismo tiempo, motivó la realización de estudios en universidades del país, que vinieron a confirmar la ocurrencia de dicho fenómeno.

Según Barrantes (1996), para la década de los 80, Estados Unidos era considerado un país con una legislación avanzada en esa materia. 
En esto contribuyó la aprobación del título viI de la Ley de Derechos Civiles en 1964, en medio de las luchas por los derechos civiles que reivindicaban a las personas afroamericanas. Posteriormente, una de las primeras organizaciones feministas, la Organización Nacional de las Mujeres (Now por sus siglas en inglés), logra que dicha legislación se amplíe, incorporando la discriminación por motivos de sexo. El argumento principal era que las proposiciones sexuales que sufrían las mujeres en el espacio laboral, atentaban contra un trato igualitario entre ambos sexos. Los primeros magistrados que conocieron estas demandas, consideraban que las "situaciones de acoso eran problemas personales entre trabajadores", por tanto, el hostigamiento era visto como un asunto trivial, producto de conductas "naturales".

Más adelante, con el advenimiento de la teoría sexo-género, la teoría feminista, los movimientos sociales de gays $y$ lesbianas, junto con otras tesis en torno al estudio de la sexualidad, se cuestiona la constitución de las identidades sexuales masculinas y femeninas a partir de la naturaleza, para dar lugar a explicaciones más de tipo social y cultural. Estas posiciones contribuyeron a "desnaturalizar" el hostigamiento sexual.

Anteriormente, la sexualidad humana era considerada como algo "natural" determinada por la biología. Esta posición denominada "esencialista", de corte fisiológico, le atribuye a la genética y a las hormonas lo que somos como seres sexuados. Algunas de estas ideas "esencialistas" fueron desarrolladas por sexólogos del siglo XIx, quienes abordaron el estudio de la sexualidad desde una visión médica. (Weeks, 1985). Estos coinciden en lo que llamaron la "naturaleza instintiva de la sexualidad", definiendo el sexo "como un impulso irrefrenable en el individuo, como una ley fisiológica o como una fuerza engendrada en poderosos fermentos que actúan como garantes de nuestro más sentido del yo" (p. 139).

Ante esta posición, se plantea la tesis "constructivista de la sexualidad", la cual sostiene que el componente biológico de la sexualidad no adquiere significados en sí mismos, sino que se construyen en las relaciones sociales. Una de sus teóricas es Rubin (1989), quien sostiene que la sexualidad no está solamente determinada por la biología, ya que se construye en la sociedad y en la historia. Agrega, que "el cuerpo, el cerebro, los genitales y el lenguaje son todos necesarios para la sexualidad humana, pero no determinan ni sus contenidos, ni las formas concretas de experimentarlo, ni sus formas institucionales" (p. 132).

Otro elemento aportado por la teoría feminista de esa época y aún vigente, apunta a que la sexualidad femenina es un tema político. Al respecto, MacKinnon (1995) afirma que "la sexualidad femenina es relacional, constructora de poder $y$ construida por el poder. El rol sexual masculino es intrusivo y agresivo hacia aquellas personas con menor poder" (p. 269). En este sentido, reconoce la existencia de una jerarquía de género en la que el deseo masculino se expresa a través de manifestaciones de violencia sexual, como la violación, el abuso, el acoso sexual y la prostitución.

Todo este desarrollo deconstructivo $y$ constructivo en torno a la sexualidad humana, aunado a la teoría feminista de la época, van permeando a la sociedad costarricense sobre esta problemática. Con el avance en materia de derechos humanos, Costa Rica llega a suscribir una serie de convenciones internacionales. En materia de hostigamiento sexual, se pueden citar: la Convención sobre la Discriminación en el Empleo y Ocupación de la Organización Internacional del Trabajo (оIт), aprobada en 1958; la Convención sobre la Eliminación de Todas las Formas de la Discriminación contra la Mujer (CEDAW), de 1979; y la Convención Interamericana para Prevenir, Sancionar y Erradicar la Violencia contra la Mujer "Convención Belem Do Para" de 1995. En particular, las dos últimas reconocen el hostigamiento sexual como un acto de violencia contra las mujeres.

La adscripción de Costa Rica a las convenciones antes citadas, obliga al país a adoptar medidas, entre ellas, aprobar o modificar leyes para desestimular y combatir el hostigamiento sexual. En este marco, es que en el año 1992, la diputada Flory Soto Valerio presentó un proyecto de ley a la Asamblea Legislativa, en el que describía el hostigamiento sexual como "parte de una extensa gama de expresiones violentas 
que por muchos siglos ha caracterizado las relaciones de subordinación especialmente entre varón y mujer" (Barrantes, 1996, 157).

Esta iniciativa no tuvo la acogida esperada $y$, para 1994, se presenta un nuevo proyecto, la exposición de motivos señala que:

...esta conducta se encuentra muy arraigada en la sociedad costarricense y constituye un impedimento para el desarrollo integral de las víctimas. Sitúa al hostigamiento como una violación a los derechos de dignidad, libertad, intimidad, trabajo, educación y desarrollo como una discriminación en razón de sexo y una violencia de género (Barrantes, 1996, p. 159).

Finalmente, en el año 1995, se aprueba la Ley nro. 7476 denominada Ley contra el hostigamiento sexual en el empleo y la docencia, que define el hostigamiento sexual "como toda conducta sexual indeseada por quien la recibe, reiterada $y$ que provoque efectos perjudiciales en los siguientes casos: condiciones materiales de empleo o de docencia, desempeño y cumplimiento laboral o educativo, estado general de bienestar personal" (Ulate, 2006, p. 40).

\section{PRIMERAS INVESTIGACIONES SOBRE HOSTIGAMIENTO SEXUAL REALIZADAS EN COSTA RICA}

A partir de la década de los 90 y en adelante, tiene lugar una serie de investigaciones en materia de hostigamiento sexual. A continuación, se presenta el cuadro 1 con información sobre el número de investigaciones por década hasta el año 2019.

CUADRO 1

INVESTIGACIONES REALIZADAS EN UNIVERSIDADES PÚBLICAS COSTA RICA, 1990-2019

\begin{tabular}{lccc}
\hline UNIVERSIDAD & DÉCADA 1990 & DÉCADA 2000 & DÉCADA 2010 \\
\hline Universidad de Costa Rica & 3 & 7 & 2 \\
Universidad Nacional & 1 & 0 & 2 \\
Universidad Costa Rica- Univ. Nacional & 1 & 3 & 1 \\
Universidad Estatal a Distancia & 0 & 2 & 1 \\
\hline
\end{tabular}

Fuente: Elaboración propia con base en las investigaciones revisadas.

Las primeras investigaciones sobre hostigamiento sexual en el país, realizadas desde la academia, datan de finales de la década de los 80 e inicios de los 90. Estas presentan la particularidad de ser trabajos anteriores a la aprobación de la citada Ley, las cuales se interesan por conocer el "estado de la cuestión" desde dos dimensiones; por un lado, desde el problema social propiamente dicho, al tratar de indagar su incidencia $y$, por el otro, desde los vacíos legales existentes para sancionar o castigar el hostigamiento en el país. A continuación, se describen algunas de estas investigaciones.
A finales de la década de 1980, el trabajo de Miranda citado por Salas (1996), es el primero sobre la materia realizado en Costa Rica y también el primero en un espacio académico. Este tenía como objetivo, precisar la magnitud de esa problemática dentro de la Universidad de Costa Rica. La población objetivo de este trabajo fueron estudiantes, docentes y personal administrativo.

Algunas conclusiones que aporta este estudio, es que los tres estamentos consideraron el hostigamiento sexual como un problema presente en el ámbito universitario. Fueron 
las mujeres quienes mayormente afirmaron lo frecuente que era en la institución. Las formas en que este se manifestaba fueron con miradas hacia el cuerpo y los piropos. Entre las razones señaladas como factores de acoso fueron el hecho de ser mujer joven, soltera, atractiva, coqueta y provocadora, así como de principios morales dudosos. Como puede observarse, este pensamiento de finales de la década, está cargado de sesgos morales y sexistas que responsabilizan a las mujeres de la provocación de tales prácticas.

En el estudio de Zavala (1992), se interesa por mostrar la frecuencia con que ocurría el fenómeno en trabajadoras del sector público y privado. De igual manera, las mujeres consideraron que era un problema grave en su espacio laboral. Sobre el origen o la causa del hostigamiento sexual, mencionaron el ser una mujer bonita o simpática, por la forma de vestir y por ser madre soltera. Algunas de las mujeres que habían sido acosadas, tenían una condición de inferior jerarquía con respecto al acosador y el rechazo a estos actos tuvo consecuencias negativas en su trabajo como por ejemplo, el cambio a un puesto de menor categoría.

Desde una perspectiva jurídica, se puede citar el trabajo realizado por Charpentier en 1993, citado por Salas (1996). En este se hace una revisión de los Códigos de Trabajo, del Código Civil, del Código Penal y la Ley General de Administración Pública, para concluir que la problemática del acoso sexual estaba regulada de manera deficiente en la normativa nacional y que además tenía un ámbito de aplicabilidad restringido, ya que solamente cubría a personas trabajadoras del sector público.

En una línea similar, se encuentra el trabajo realizado por Alvarado en 1993, citado por Salas (1996), en el cual se hace una revisión más acotada del Código de Trabajo, para afirmar que la regulación del hostigamiento era muy "tímida" y que no brindaba la suficiente protección a las mujeres trabajadoras, por lo que recomendaba para subsanar esa "debilidad", la creación de una ley especial. De igual manera, la revisión de los códigos nacionales efectuada por Solano y Badilla en 1993, citados por Salas (1996), llega a conclusiones muy parecidas a la anterior, en cuanto confirma la desprotección de las trabajadoras costarricenses ante el hostigamiento sexual.

La investigación de Barrantes (1996), resalta la importancia del trabajo realizado por Obando en 1994, en dos sentidos; por un lado, destaca la visión moralista de la legislación vigente en materia de delitos sexuales $y$, por otro, presenta una propuesta de un proyecto de ley que más adelante sería la base de la Ley nro. 7476, que obliga a las instituciones públicas del país a disponer de un reglamento para sancionar el hostigamiento sexual.

Los resultados de los trabajos anteriores mostraron que el hostigamiento sexual estaba ocurriendo en los espacios de estudio $y$ de trabajo donde se investigó. Esto es importante al mostrar la existencia de un "problema" que hasta ese momento era invisible y desconocido, el cual evidenciaba que eran las mujeres quienes más lo sufrían, que la percepción del fenómeno estaba atravesada por mitos y estereotipos expresados por las mujeres sujetas de estudio, al considerar a sus congéneres como culpables o provocadoras de estos actos. También se constata que la normativa existente en el país era insuficiente para la protección a las mujeres. Esta información constituye los antecedentes para la propuesta de un proyecto que culmina con la aprobación de la Ley nro. 7476.

También, estas investigaciones tienen en común un abordaje teórico que parte de la construcción social de las identidades masculinas y femeninas. Da importancia a los procesos de socialización, en los cuales cada sociedad asigna unos roles y funciones que debe asumir cada sexo. Estos roles de género crean comportamientos y prescripciones sociales para cada uno de ellos. En relación con la sexualidad para los hombres, se construye una sexualidad más permisiva $y$ libre, bajo un estereotipo de un deseo sexual siempre activo, mientras que para las mujeres la sexualidad se destaca más por su función reproductiva. 
INVESTIGACIONES SOBRE HOSTIGAMIENTO SEXUAL POSTERIORES A LA APROBACIÓN DE LA LEY NRO. 7476

La primera investigación que tiene lugar, una vez aprobada la citada ley, es realizada por Salas (1996), en el Instituto Costarricense de Electricidad (ICE) ${ }^{2}$. Sus actividades como funcionaria del ICE, la llevaron a tener contacto con mujeres secretarias y en otros cargos administrativos, quienes le expresaron su inquietud sobre situaciones de hostigamiento sexual que estaban experimentado. Esto llevó a la realización de un sondeo que vino a confirmar las vejaciones que estaban sufriendo algunas mujeres dentro de la Institución. También fue la base para la aprobación posterior de una modificación del Estatuto de Personal de la Gerencia, para que los actos de hostigamiento o acoso sexual fueran considerados faltas graves $y$, a su vez, fueran sancionados con despido sin responsabilidad patronal.

Estos hechos acaecidos en el año 1994, son considerados, desde la perspectiva de la autora, hitos históricos, ya que el ICE, fue la primera empresa gubernamental a nivel de Centroamérica en aprobar una normativa para sancionar el hostigamiento sexual. Este evento contribuyó a impulsar la legislación para sancionar el hostigamiento sexual en Costa Rica, con lo que se convirtió en un país pionero a nivel de América Latina en aprobar una ley específica en el año 1995, como ya se mencionó.

El trabajo de esta tesis se interesó por determinar el significado psicosocial que las mujeres le asignaban al hostigamiento sexual, pero también consideró la participación de algunos hombres. La concepción del hostigamiento mostró diferencias entre sexos, ya que los hombres manifestaron que las víctimas de hostigamiento sexual eran mujeres que "provocan" $y$ asociaron el acoso con "cortejo", al mismo tiempo que los acosadores eran vistos como "hombres enfermos". Este tipo de pensamiento, es coincidente con los hallazgos del trabajo

$2 \quad$ El Instituto Costarricense de Electricidad (ICE) es el ente estatal público en Costa Rica que brinda los servicios de electricidad $y$ telecomunicaciones, fue fundado en 1949. de Wise y Stanley (1992) y, a su vez, reafirma la concepción estereotipada de la sexualidad masculina $y$ de igual manera la femenina, que deviene de siglos atrás, la cual se consideraba determinada por la naturaleza y los instintos.

La investigación realizada por Picado et al. (1997), fue la primera en realizarse en la Universidad Nacional. Esta se plantea el objetivo de conocer sobre el estado de la reglamentación de la Ley nro. 7476, en algunos hoteles del área metropolitana. Igualmente, se pretendió indagar qué estaban haciendo las jefaturas en situaciones de denuncia. El trabajo destaca la poca claridad que se tenía sobre qué podía considerarse como hostigamiento sexual. En este aparecen las mujeres como las más afectadas, siendo los acosadores, hombres en condición de jefatura y clientes del hotel. Se constató que un alto porcentaje de las personas no conocían la existencia de la Ley que sanciona el hostigamiento sexual.

Finalizando la década de los 90, se realizó una investigación con un grupo de enfermeras del hospital Dr. Tony Facio de Limón, a cargo de Elizondo y Padilla (1999). De igual manera, un alto porcentaje de las enfermas no tenían conocimiento de la recién aprobada Ley. El hostigamiento sexual se manifestaba a través de acercamientos corporales, palabras con contenido sexual, piropos y miradas lascivas. Las mujeres eran las principales víctimas y los acosadores habían sido médicos, pacientes, visitantes $y$ otros profesionales en enfermería. Los actos ocurrieron en mayor medida en horario nocturno. La irritabilidad, el desgano y la frustración fueron las consecuencias más sentidas por las mujeres.

Las investigaciones realizadas en este período tienen en común que se continua con la percepción sobre el hostigamiento sexual cargada de mitos y de estereotipos sexistas. A cinco años de haberse aprobado la Ley, las personas indagadas tenían poco conocimiento sobre esta. Sin embargo, se logra confirmar la existencia del problema en las instituciones donde tuvo lugar la investigación. Reafirma que las víctimas son mayormente las mujeres y las prácticas de acoso provienen de los hombres indistintamente de la condición jerárquica; las 
manifestaciones se expresan en miradas lascivas, tocamientos corporales, insinuaciones, piropos, entre otros.

La década que inicia en el año 2000, viene aparejada por un mayor interés por investigar el hostigamiento sexual en el país, prueba de ello es que se registran un mayor número de trabajos. La investigación realizada por las abogadas Alfaro y Montero (2000), plantea que la Ley nro. 7476, no contenía una especificación adecuada sobre cómo debía aplicarse la sanción por hostigamiento sexual para funcionarios públicos que han llegado al poder mediante la elección popular. Para resolver esta omisión, proponen reformar algunos artículos a través de un nuevo proyecto que aplica para puestos de presidente $y$ vicepresidente de la República, diputados, diputadas y munícipes. Las autoras consideran que los puestos antes citados, no deben de gozar de un fuero especial, ya que se estaría afectando el principio de igualdad que debe regir para las personas funcionarias como principio constitucional. Discrepan en que deba ser el Tribunal Supremo de Elecciones, el órgano competente para sancionar situaciones de este tipo, en su lugar consideran que deben ser los tribunales laborales.

La revisión de reglamentos para determinar su efectividad en la sanción del hostigamiento sexual, fue el interés de Saravia y Saravia (2002). Las autoras efectuaron una revisión de los reglamentos del Instituto Costarricense de Electricidad, el Instituto Nacional de Aprendizaje y el banco privado Banex. Concluyeron que las pocas denuncias presentadas en esas instituciones fueron sancionadas con despidos sin responsabilidad patronal $y$, en otros casos, se aplicaron sanciones menos severas. Algunas denuncias se resolvieron en el tiempo establecido para ello, que es de tres meses, mientras que otras demoraron un poco más de tiempo. Para finalizar afirman que en estas instituciones se estaba aplicando el debido proceso en la resolución de las denuncias.

Determinar el conocimiento que se tenía sobre la problemática del hostigamiento sexual en instituciones educativas fue el interés de Fuentes (2003). Para ello tomó en cuenta 10 escuelas rurales del cantón de San
Ramón de Alajuela. Los resultados mostraron que directores, directoras, docentes y estudiantes tenían poco conocimiento sobre la ocurrencia del hostigamiento sexual en las escuelas, tampoco conocían la existencia de un reglamento ni el procedimiento que sanciona estos actos a nivel de la institución. Un elemento positivo fue que las tres poblaciones tenían noción sobre qué podían considerarse actos de acoso sexual cuando se les presentaron algunas manifestaciones.

El seminario de graduación llevado a cabo por Chacón et al. (2003), tuvo como objetivo determinar qué conocimientos tenían un grupo de estudiantes, docentes y personal administrativo de la Sede de Occidente de la Universidad de Costa Rica. Las tres poblaciones coincidieron en reconocer como hostigamiento sexual aquellas actitudes hostiles e indeseadas que afectan la integridad física, psicológica y sexual de las personas. Se encontró que las acosadas eran principalmente mujeres estudiantes por parte de hombres docentes, en mayor grado, en condición de interinos. Continúa presente el mito que considera que la persona hostigada provoca el acoso sexual, para ser más preciso, es provocado por las mujeres.

La investigación llevada a cabo por Carvajal (2004), se realizó con población estudiantil de la Universidad Nacional. Tuvo como objetivo, conocer la prevalencia, las manifestaciones y los efectos del hostigamiento sexual, al utilizar para ello 16 manifestaciones clasificadas en las categorías: leve, moderada, grave $y$ muy grave. Los resultados de este trabajo mostraron una diferencia entre la percepción y la manifestación del acoso sexual.

A la pregunta de si había sufrido hostigamiento la mayoría del estudiantado se respondió de manera negativa, pero cuando se le presentan las manifestaciones, la respuesta se tornó afirmativa. La mayor parte de las personas consideraron que habían sido acosadas de manera leve, al haber recibido exclamaciones, silbidos insinuantes $y$ se sintieron desnudadas o desnudados con la mirada. En estos casos, el hostigamiento provino de sus pares o compañeros. Otra parte consideró el acoso recibido de manera grave, ya que recibieron invitaciones a 
cenar o tomar, fueron tocadas en alguna parte del cuerpo y les ofrecieron aprobar un curso o mejorar la nota a cambio de sexo. Estos actos fueron cometidos por profesores. Los efectos causados presentan diferencias por sexo, en las mujeres produjeron enojo, vergüenza, miedo, irritabilidad e inseguridad, mientras que en los hombres, de igual manera produjeron enojo $y$ vergüenza, pero en porcentajes menores.

Una investigación similar fue la realizada por Guzmán (2005), en la Universidad de Costa Rica, con una población que incluía estudiantes, docentes, personal administrativo y autoridades. Esta tenía como objetivo determinar qué conocimientos, actitudes y prácticas existían en torno al acoso sexual a 10 años de haberse aprobado la respectiva Ley. Los resultados fueron similares a trabajos anteriores, en la que las mujeres aparecían como las más acosadas, siendo el acosador un hombre. También aparecieron hombres acosados por otros hombres. Las prácticas ocurrieron mayormente en actividades académicas y laborales, en lugares solitarios del campus y sin presencia de otras personas. Los efectos causados fueron intranquilidad, enojo, desmotivación y frustración. Subsiste el mito de que la persona hostigada provoca esa conducta, ya sea por su forma de vestir o de comportarse.

En una línea similar, Ulate (2006) realiza su investigación con la finalidad de conocer el funcionamiento del reglamento que sanciona el hostigamiento sexual en la Universidad $\mathrm{Na}$ cional, a 10 años de vigencia. Particularmente, analiza la efectividad del mecanismo y los obstáculos que se han encontrado para su aplicación. Uno de los principales hallazgos, producto de la revisión de 10 expedientes, es que los procesos han excedido el tiempo reglamentario estipulado de 3 meses, en todos los casos el período ha oscilado entre los 100 y 600 días. Esta "morosidad" también se encontró en 5 denuncias que estaban pendientes de resolver. Para la autora, esta situación no solo contraviene los derechos de las personas denunciantes, que en estos casos eran mujeres estudiantes, sino que además estos largos procesos generan desgaste físico y emocional. La explicación a esa lentitud es el exceso de tecnicismos utilizados por la parte denunciada $y / o$ del Tribunal Universitario de Apelaciones, tales como, repetición de audiencias, sobreutilización de recursos de apelación, todo ello con la finalidad de dilatar el proceso de apertura o la resolución final.

Otro aspecto analizado fueron las sanciones impuestas a los 10 casos estudiados, sobre este particular la investigadora considera, que en algunos, se ha dado una errónea aplicación de la legislación nacional e internacional en materia de acoso sexual:

...cuando las sanciones son leves para la falta cometida, cabe la sospecha que el deseo de las autoridades juzgadoras era proceder con benevolencia con el denunciado, mientras que, para el sujeto discriminado por la sociedad la autoridad no compensó el déficit de sus legítimos derechos (Ulate, 2006, p. 65).

La vivencia del acoso sexual por mujeres policías fue el interés del trabajo realizado por García (2006). Los tocamientos de pechos, de sus genitales, abrazos, palabras obscenas e invitaciones a salir, fueron los actos cometidos por hombres en condición de jerarquía. Las situaciones de acoso ocurrieron, por lo general, a altas horas de la noche, cuando ellas se encontraban solas, lo que dificultó posteriormente la credibilidad de los hechos denunciados. La autora considera que, ante la ausencia de testigos, prevalecen los mitos que le acreditan a la denunciante cierta responsabilidad y el consentimiento de los hechos.

Asimismo, se tiende a desvalorizar o subestimar los hechos denunciados "al no existir indicios suficientes", se termina calificando "como faltas de respeto para la víctima", ante lo cual las sanciones para los acosadores son menos drásticas. Las policías coincidieron en que la denuncia del acoso tuvo consecuencias en las condiciones laborales, como la asignación de trabajos considerados "feos" o sufrir recargo de labores, traslado a otro lugar de trabajo, no ser tomadas en cuenta en actividades de capacitación o la no asignación del arma reglamentaria, pero el temor mayor era perder el trabajo, que es el medio económico de subsistencia. Estas situaciones produjeron 
ansiedad, desconcentración y baja autoestima, lo que llevó a tomar medicación antidepresiva $y / 0$ ansiolíticos.

Un trabajo similar se realizó 10 años después en la Escuela Nacional de Policía del Ministerio de Seguridad Pública por Arce y Rodríguez (2017). Las autoras consideran que la relación jerárquica que se establece entre el alumnado y el personalinstructor en la Escuela favorece la existencia de prácticas de hostigamiento sexual.

Otra institución objeto de estudio fue el Poder Judicial a través del trabajo realizado por Rodríguez (2008). En este se concluye que a las personas trabajadoras de ese Poder, desconocían dónde interponer una denuncia por hostigamiento sexual, a la vez que lo consideraron un problema grave en la Institución.

El trabajo realizado por Torres (2009), reconstruye lo acontecido en un Colegio de Liberia, cuando unas estudiantes menores de edad manifestaron estar recibiendo insinuaciones $y$ comentarios con contenido sexual, piropos $y$ miradas por parte de un profesor. La autora concluye que hubo un inadecuado manejo del problema por parte de las autoridades, que condujo a que el proceso se alargara por varios años, lo que ocasionó re-victimización a las estudiantes, la reprobación del curso, un desgaste emocional severo, la generación de ideas suicidas, desórdenes alimenticios e ingesta de licor, entre otros. Rosales (2009), efectúa una revisión de los reglamentos y de los procedimientos que estaban aplicando las cuatro universidades públicas para sancionar el hostigamiento sexual. Concluye que las entidades han establecido estructuras diferentes para conocer $y$ sancionar las faltas por este motivo.

Valverde (2010), muestra cómo opera la Ley nro. 7476 en situaciones donde no impera una relación obrero-patronal. La autora plantea que en la contratación laboral bajo la modalidad outsourcing ${ }^{3}$ y servicios profesionales,

$3 \quad$ Outsourcing es un término del inglés que se traduce al español como "subcontratación". Es un proceso en el cual una institución, organización o empresa, contrata a otras empresas externas para que realicen una parte de su actividad o producción. deben darse una serie de responsabilidades entre la empresa contratante y la prestadora del servicio para evitar la ocurrencia del hostigamiento sexual. Entre estas se encuentra sentar las responsabilidades de previo a la firma del contrato. Otra sería solicitar a la empresa contratada que incluya entre sus políticas, el principio de ambiente libre de hostigamiento sexual. En el caso de la contratación por servicios profesionales, sugiere agregar al contrato, una copia del reglamento interno de la empresa que previene y sanciona el hostigamiento sexual e, incluir, una cláusula indicando que se abstenga de cometer este tipo de actos.

Un nuevo trabajo con población estudiantil de la Universidad Nacional fue realizado por Carvajal y Delvó (2010), el cual complementa el efectuado por Carvajal en el 2004 — citado anteriormente - con lo cual se pudo realizar comparaciones. Estos nuevos datos vienen a confirmar que en este centro de estudios continúan siendo las mujeres las más afectadas por el hostigamiento sexual, aunque en menor porcentaje con respecto al trabajo anterior. Los efectos causados fueron muy similares, ya que se repitió el enojo, el miedo, la inseguridad y la irritabilidad. En cuanto a las reacciones, las mujeres optaron por el silencio, $y$ aunque les causó molestia, algunas llegaron a comentarlo con alguien de confianza. Mientras que en los hombres, la reacción más frecuente fue no darle importancia o tomarlo a broma.

Las investigadoras Mora y Matteucci (2010), hacen una propuesta para fortalecer las capacidades de las personas que integran las comisiones instructoras, que son las responsables de investigar las denuncias sobre hostigamiento sexual en la Universidad de Costa Rica. Elaboran un manual de autoformación para que dichas personas dispongan de herramientas para un mejor abordaje de esta problemática. Para ello, proponen seis ejes temáticos, que incluyen aspectos sociales, legales y psicológicos sobre el hostigamiento sexual.

Otro esfuerzo por visibilizar la problemática del acoso en el lugar de trabajo fue el realizado por Marín (2012). Este se interesó por conocer la prevalencia $y$ las manifestaciones en el Instituto Nacional de Aprendizaje (INA). Un 
mayor porcentaje de mujeres que hombres, reconocieron haber sido víctimas de acoso sexual, este provenía de hombres estudiantes quienes hicieron comentarios, ruidos y gestos con connotación sexual que resultaron molestos. Las mujeres expresaron haber sentido vergüenza por el temor a que se viera juzgada su sexualidad o que pudiera pensarse que fue un acto provocado o consentido por ellas. Se encontró que cuando el hostigamiento ocurre dentro de una relación jerárquica, esta adquiere características de chantaje, ya que se ofrece algo a cambio de un favor sexual, en estos casos aparecieron involucrados los docentes de los cursos.

Una nueva investigación de las autoras Carvajal y Delvó (2012), se realizó con población administrativa de la Universidad Nacional. Para ello, utilizaron un listado de manifestaciones, entre las que se contaban las exclamaciones o los silbidos insinuantes, acercamientos corporales, haber recibido miradas perturbadoras, recepción de llamadas telefónicas o mensajes de texto con contenido sexual. Los resultados reiteran la afectación de un mayor número de mujeres. La persona hostigadora fue un compañero de trabajo en la mayoría de las veces, aunque también aparecen mujeres $y$ jefes. En el caso de los hombres hostigados, otros hombres y mujeres aparecen como hostigadores. Sobre los efectos, aparecen nuevamente el enojo, el miedo, la inseguridad y la vergüenza, estos más presentes en mujeres que en hombres. Es reiterada la tendencia de las mujeres a no decir nada o solamente comentárselo a otra persona de confianza, y en el caso de los hombres a no darle importancia.

Las investigaciones realizadas posteriormente a la aprobación de la Ley nro. 7476, también han sido de utilidad para evidenciar la existencia u ocurrencia del hostigamiento en las universidades e instituciones donde se realiza el trabajo. Con estas se logra evidenciar $y$ mostrar un problema social oculto o invisibilizado, se reitera que son las mujeres las más acosadas por parte de hombres, así como muestran las diversas formas en que este se manifiesta $y$ dan algunas pautas sobre los lugares y las horas en las que las mujeres se vuelven más vulnerables a sufrir el acoso. Además, se muestra la afectación en la salud física y emocional de las mujeres que lo sufren. Evidencian la prevalencia de mitos $y$ estereotipos que culpabilizan a las mujeres como las provocadoras del hostigamiento. Ponen de manifiesto que los largos procesos en su resolución generan desgaste físico y psicológico a las víctimas. Muestran que subsiste desconocimiento de la existencia de una ley, aun habiendo pasado varios años de su promulgación. Las mujeres que se atreven a denunciar sufren un desmejoramiento de sus condiciones laborales que las amenaza hasta con perder su trabajo.

Cabe señalar que las investigaciones que datan aproximadamente del año 2010 en adelante, si bien, constatan que son las mujeres las más afectadas por hostigamiento sexual, agregan datos que indican que los hombres también son víctimas de acoso por mujeres y por otros hombres. Sin embargo, el problema solo se menciona y no se dan mayores detalles sobre el mismo. Sobre cómo viven el hostigamiento los hombres, es un tema pendiente de investigar.

Las investigaciones realizadas para optar por el grado de maestría, Salas (1996), Carvajal (2004), García (2006), Ulate (2006) y Marín (2012), tienen en común el abordaje teórico feminista, el cual ubica el hostigamiento sexual como un problema de poder y dominio en el marco de la sociedad patriarcal. Lerner (1990) define el patriarcado como:

...la manifestación y la institucionalización del dominio masculino sobre las mujeres y los niños de la familia y la ampliación de ese dominio masculino sobre las mujeres a la sociedad en general. Ello implica que los varones tienen el poder en todas las instituciones importantes de la sociedad y que se priva a las mujeres de acceder a él (Lerner, 1990, pp. 340-341).

Los trabajos de García y Bedolla, publicados en 1993, también son citados en varios de estos estudios. Para estas autoras, el acoso sexual es considerado un acto de discriminación basado en el poder, la sexualidad y la violencia. El poder se expresa en 
el dominio de los hombres sobre la otra mitad de la población, en la cual, la victimización sexual es una expresión de este. En sociedades patriarcales es usual que las mujeres sean consideradas un objeto sexual para satisfacción de los deseos sexuales de los hombres. De ahí que el hostigamiento sea considerado un acto de agresión o de violencia sexual, y un abuso de poder basado en la jerarquía del género (Rosales, 2009).

Es importante mencionar que, a finales del año 2017, salen a las luz pública, las denuncias realizadas por un número importante de mujeres ligadas al mundo de la actuación contra el productor cinematográfico Harvey Weinstein, quienes lo acusan de hostigamiento, agresiones sexuales y violación. Estas denuncias en cascada han contribuido al surgimiento del movimiento conocido como "Me Too", que ha inspirado otras denuncias tanto en Estados Unidos como en otros países del mundo y han permeado a la sociedad costarricense.

Al momento de la elaboración de este artículo, un grupo de mujeres estudiantes de las universidades públicas denuncian, ante la comunidad nacional, una serie de hechos de hostigamiento sexual. Por ejemplo, en la Universidad de Costa Rica y en la Universidad Nacional los movimientos "MepasoenlaUCR" y "MepasoenlaUNA", a través de las redes sociales, han denunciado hostigamiento sexual por parte de profesores, prácticas sexistas e impunidad en sus instituciones. Algunas de sus demandas apuntan a cambios en los reglamentos para aplicar sanciones más severas y mayor rapidez en la resolución de las denuncias. Estos movimientos bien pueden ser objeto de futuras investigaciones.

Esto evidencia la subsistencia del problema, aun habiendo investigaciones que confirman o constatan la existencia del hostigamiento, la existencia de una ley específica con casi 25 años de existencia, reglamentos y órganos competentes que reciben y resuelven las denuncias, además de campañas y actividades de información y sensibilización. Esto denota que toda esta estructura ha sido de utilidad para la creación de espacios de denuncia, pero ha sido insuficiente para contener la ocurrencia de dichas prácticas sexistas y discriminatorias.

Las estudiantes solicitan una declaratoria de emergencia que en alguna medida obligue a las universidades, por un lado, a hacer un balance entre lo actuado $y$ lo logrado $y$, por otro, a establecer una estrategia que desestimule la ocurrencia de estas prácticas discriminatorias. También, esto podría motivar la realización de otras investigaciones más de corte cualitativo.

Actualmente, gracias al aporte investigativo realizado desde las universidades públicas, se dispone de mayor información y conciencia que impone nuevos retos en la atención, prevención y sanción del hostigamiento sexual, pero el desafío mayor está en la erradicación de estas prácticas discriminatorias que violentan el derecho al estudio y al trabajo de muchas mujeres libre de violencia.

\section{CONCLUSIONES}

$\diamond \quad$ Las investigaciones realizadas en las universidades han contribuido a visibilizar un problema social que afectaba a la sociedad costarricense, que estaba "oculto", que la realidad sociocultural de ese momento histórico no permitía reconocerlo como tal. Han venido a generar una conciencia crítica sobre esta problemática. Con el advenimiento de este nuevo conocimiento, aunado al posicionamiento de derechos humanos, estos problemas sociales tan arraigados y normalizados por la naturaleza, comienzan a adquirir una dimensión diferente, en la cual el acoso se relaciona con prácticas discriminatorias y violentas hacia las mujeres.

$\diamond \quad$ Las universidades públicas han hecho un aporte muy valioso al conocimiento $y$ visibilización de esta compleja problemática social. Con ello, han puesto de manifiesto que el hostigamiento sexual lo sufren mayormente las mujeres y que los acosadores son principalmente los hombres. Estas conductas indeseadas causan un grave daño a la salud física y emocional de las personas, además de violentar sus derechos humanos. 
$\diamond \quad$ La mayor parte de los trabajos han sido de tipo cuantitativo, muestran cifras o datos que ayudan a confirmar y a darle veracidad a la existencia del hostigamiento sexual como prácticas entronizadas en las instituciones donde se realizaron los estudios. A partir de los mismos, se convierten en la base para proponer acciones, así como para desestimular, combatir y sancionar el hostigamiento sexual. Se presenta una búsqueda de construcción de espacios de trabajo $y$ estudio, libres de estas indeseadas prácticas.

$\diamond \quad$ Las investigaciones también han contribuido a disipar las "ambigüedades" que en algún momento caracterizaron las prácticas de hostigamiento sexual. Estas han logrado visibilizar que las más afectadas son las mujeres $y$ que son los hombres los que mayormente incurren en estas prácticas. Asimismo, se demuestran las diversas formas en qué ocurre $y$ la manera en que afecta a las personas. Los estudios también han sido de utilidad para confirmar la prevalencia o existencia de mitos y estereotipos explicativos sobre el origen del hostigamiento sexual. Esto conlleva a la realización de mayores esfuerzos por generar una "ruptura epistemológica", que involucra a las personas afectadas por el hostigamiento sexual, a las que atienden e investigan las denuncias en las instituciones $y$ a las autoridades universitarias que emiten resoluciones. En esta línea deben continuarse los esfuerzos de información, sensibilización y capacitación de deconstrucción, a partir de la revisión de la construcción de las masculinidades $y$ feminidades.

$\diamond \quad$ Algunos trabajos más recientes dan cuenta que también los hombres están siendo afectado por este problema; no obstante, es poca la información que se aporta. Este podría ser un tema para considerar en futuras investigaciones.

$\diamond \quad$ Las reacciones diferentes que asumen mujeres $y$ hombres ante conductas de acoso son de vital relevancia. En el caso de las mujeres, la conducta reiterada de una posición pasiva y poco contestaría ante situaciones de acoso merece atención. De igual manera, en el caso de los hombres, quienes dan poca importancia o toman a broma las conductas de hostigamiento recibidas. En ambos casos, se hace necesario investigaciones cualitativas que expliquen esos comportamientos $y$ actitudes.

$\diamond \quad$ Las diferencias encontradas sobre el reconocimiento del hostigamiento sexual ante una pregunta directa versus sus manifestaciones, evidencian cómo está naturalizado el hostigamiento sexual en nuestra sociedad, con lo cual se deben considerar en futuras investigaciones, en el diseño de campañas de información y divulgación. Las manifestaciones suelen ser reconocidas e identificadas con mayor facilidad $y$ pueden ser la base de campañas de sensibilización e información.

$\diamond \quad$ Es importante destacar el papel que han jugado las oficinas, centros o institutos de género, o de la mujer, de las universidades $y$ de algunas instituciones, en asumir un papel protagónico en la atención de esta problemática. Se han interesado en la realización de campañas informativas para la prevención, han incentivado el reconocimiento de las manifestaciones e incitado a la denuncia. Cabe hacer una reflexión en torno a si deberían continuar ejerciendo ese papel o, estas funciones deban ser asumidas por cada universidad.

\section{REFERENCIAS}

Alfaro, G. y Montero, M. (2000). El hostigamiento sexual en puestos de elección popular. [Trabajo final de graduación para optar por el título de Licenciado en Derecho]. Universidad de Costa Rica.

Arce, A. y Rodríguez, K. (2017). Acoso sexual en la Escuela Nacional de Policía del Ministerio de Seguridad Publica en su sede central durante el cuarto trimestre del 2016. [Trabajo presentado para optar al título de Magister]. Universidad Estatal a Distancia. 
Barrantes, E. (1996). Acoso sexual: análisis de derecho comparado entre la legislación costarricense y la legislación estadounidense. [Tesis para optar por el grado de Licenciada en Derecho]. Universidad de Costa Rica.

Carvajal, Z. (2004). Prevalencia, manifestaciones y efectos del hostigamiento sexual en la Universidad Nacional. [Tesis para optar al grado de Maestría]. Universidad Nacional y Universidad de Costa Rica.

Carvajal, Z. y Delvó, P. (2010). Universidad Nacional: reacciones $y$ efectos del hostigamiento sexual en la población estudiantil en el 2008. Revista de Ciencias Sociales, 126-127, 59-74.

Carvajal Z. y Delvó P. (2012). Hostigamiento sexual en el sector administrativo de la Universidad Nacional: prevalencia, manifestaciones y efectos. Revista Feminista Casa de la Mujer, 21 (1), 25-66.

Chacón, R., Herrera, A. M., Pérez, M. A. y Villalta, G. (2003). El hostigamiento sexual en la Universidad de Costa Rica, Sede de Occidente. Recinto de San Ramón, en el período 2001-2002 y la intervención del o la profesional en trabajo social para la prevención y atención del mismo como fenómeno social. [Memoria de seminario de graduación para optar por el título de Licenciatura]. Universidad de Costa Rica.

Elizondo, G. y Padilla, P. (1999). El hostigamiento sexual, un estudio situacional de la problemática en la profesión de enfermería del Hospital Dr. Tony Facio Castro, durante el mes de mayo 1999. [Tesis para optar por el grado de Licenciatura]. Universidad de Costa Rica.

Fuentes, F. (2003). La aplicación de la Ley contra el hostigamiento sexual en la gestión de los directores de enseñanza primaria de Circuito 02 de la Dirección Regional de San Ramón. [Informe final para optar por el grado de Máster]. Universidad de Costa Rica.

García, E. (2006). El acoso sexual como politica de terrorismo: experiencia de las mujeres policías funcionarias de la fuerza pública de Costa Rica. [Trabajo final para optar a la Maestría]. Universidad de Costa Rica y Universidad Nacional.

García, B. y Bedolla, P. (1993). Las relaciones de poder $y$ violencia vinculadas al hostigamiento sexual. En P. Bedolla, (Ed.), Estudios de género y feminismo II. Universidad Nacional Autónoma de México.

Guzmán, L. (2005). Prevención del Hostigamiento Sexual en la Universidad de Costa Rica. Informe Final. Proyecto de InvestigaciónAcción. San José, Costa Rica.

Lerner, G. (1990). La creación del patriarcado. Editorial Crítica S.A.

MacKinnon, C. (1979). Sexual Harassment of Working Women: A case of sex discrimination. Yale University Press.

MacKinnon, C. (1995). Hacia una teoría feminista del Estado. Ediciones Cátedra S. A.

Marín, I. (2012). Prevalencia y manifestaciones de hostigamiento sexual en el Instituto Nacional de Aprendizaje (INA). [Tesis para optar por el grado de Maestría]. Universidad de Costa Rica.

Mora, E. y Matteucci, A. (2010). Hostigamiento sexual en la Universidad de Costa Rica: manual de autoafirmación para comisiones instructoras. [Trabajo final de graduación para optar por el grado de Licenciatura]. Universidad de Costa Rica.

Picado, Y., Acuña, G., Bustos, J., González, M., Madrigal, L.G., Salazar, C. y Vásquez, W. (1997). El hostigamiento sexual en el empleo, un caso: el subsector hotelero. [Memoria presentada como trabajo final de graduación para optar por la Licenciatura]. Universidad Nacional.

Rodríguez, M. (2008). Cumplimiento de políticas preventivas y sancionatorias con perspectiva de género en torno a la ley contra el hostigamiento sexual en el empleo y la docencia por parte del poder judicial de 1996 a 2007. [Tesis para optar al grado de Maestría]. Universidad Estatal a Distancia.

Rosales, M. (2009). Hostigamiento sexual en la docencia: estudio comparativo de los reglamentos internos contra el hostigamiento sexual y los procedimientos 
seguidos en las instituciones educativas superiores públicas. [Tesis para optar al título de Licenciatura]. Universidad de Costa Rica.

Rubin, G. (1989). Reflexionando sobre el sexo: notas para una teoría radical de la sexualidad. En Carol Vance (comp), El placer y el peligro, explorando la sexualidad femenina (pp. 113-190). Editorial Revolución S.A.L.

Salas, M. (1996). Los significados psicosociales del acoso sexual a las mujeres en el ambiente de trabajo: el caso del ICE. [Tesis para optar al grado de Máster]. Universidad de Costa Rica y Universidad Nacional.

Saravia, M. y Saravia, A. (2002). Eficacia y aplicabilidad de la ley contra el hostigamiento sexual en el empleo y la docencia. Análisis de los procedimientos sancionatorios y de la posibilidad real de su aplicación a nivel nacional. [Tesis para optar por el título de Licenciatura]. Universidad de Costa Rica.

Torres, T. (2009). El hostigamiento sexual en un centro educativo de la ciudad de Liberia: la experiencia de dos mujeres adolescentes que denunciaron a un profesor en el 2006. [Tesis para optar el grado de Licenciatura]. Universidad de Costa Rica.

Ulate, C. (2006). Ley contra el hostigamiento sexual en el empleo y la docencia. Problemas en su aplicación: el caso de la Universidad Nacional. [Tesis para optar al grado de Maestría, Universidad Nacional-Universidad de Costa Rica].

Valverde, A. (2010). El procedimiento sancionatorio por hostigamiento sexual cuando involucra sujetos vinculados a empresas del sector privado mediante formas de contratación no laboral. [Tesis para optar por el título de Licenciatura]. Universidad de Costa Rica.

Weeks, J. (1985). El malestar de la sexualidad. Significados, mitos y sexualidades modernas. Talasa Ediciones S.L.

Wise, S. y Stanley, L. (1992). El acoso sexual en la vida cotidiana. Paidós.

Zavala, G. (1992). Hostigamiento sexual de la mujer en el trabajo. Su problemática social y tratamiento jurídico. [Tesis para optar al grado de Licenciatura]. Universidad de Costa Rica.

Fecha de ingreso: 03/02/2020 Fecha de aprobación: 24/11/2020 
